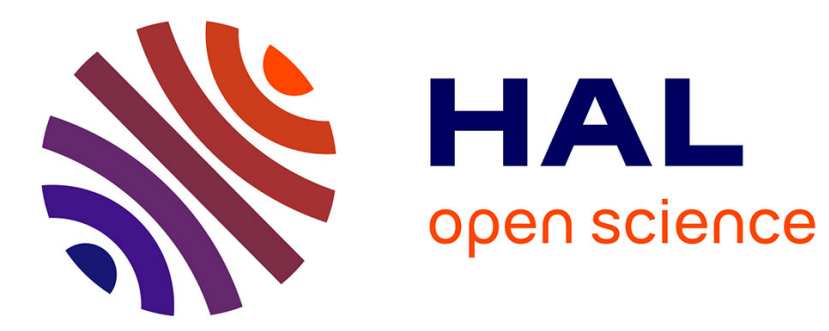

\title{
Gouverner par les incitations. La diffusion d'une logique incitative dans le droit du travail
}

Olivier Leclerc, Tatiana Sachs

\section{To cite this version:}

Olivier Leclerc, Tatiana Sachs. Gouverner par les incitations. La diffusion d'une logique incitative dans le droit du travail. Revue Française de Socio-Economie, 2015, 2, pp.171-185. 10.3917/rfse.hs1.0171 . halshs-01228816

\section{HAL Id: halshs-01228816 \\ https://shs.hal.science/halshs-01228816}

Submitted on 4 May 2020

HAL is a multi-disciplinary open access archive for the deposit and dissemination of scientific research documents, whether they are published or not. The documents may come from teaching and research institutions in France or abroad, or from public or private research centers.
L'archive ouverte pluridisciplinaire HAL, est destinée au dépôt et à la diffusion de documents scientifiques de niveau recherche, publiés ou non, émanant des établissements d'enseignement et de recherche français ou étrangers, des laboratoires publics ou privés. 


\section{Gouverner par les incitations. La diffusion d'une logique incitative dans le droit du travail}

Olivier Leclerc

CERCRID, CNRS, université de Lyon, université Jean-Monnet

olivier.leclerc@univ-st-etienne.fr

Tatiana Sachs

IRERP, université Paris Ouest-Nanterre la Défense

tatiana.sachs@u-paris10.fr

Article paru in Revue française de socio-économie, 2015/2 (hors-série), pp.171-185

DOI: $10.3917 /$ rfse.016.0171

Résumé : Certaines normes, plus que d'autres, méritent d'être qualifiées d'« incitatives » : telle est l'hypothèse qui constitue le point de départ de cet article. À rebours d'une tentation communément partagée par les juristes et les économistes à considérer que toutes les règles juridiques constituent des incitations, l'article isole les attributs des dispositifs incitatifs, afin de mieux cerner ce que la prolifération de ce type d'instruments juridiques produit dans l'ordonnancement du droit du travail. La figure de l'employeur, mais aussi l'articulation de l'individuel et du collectif s'en trouvent affectées.

Abstract : This paper builds on the idea that some norms deserve more than others to be said incentives. Unlike legal scholarship and economists who tend to hold every single legal norm as being an incentive, the paper narrows the scope of what constitutes an incentive. It then analyzes the consequences of the pervasiveness of that kind of norms in labour law. The conception of what an employer does, as well as the individual-collective nexus in labour settings, are found to be affected.

Mots clés : incitations, droit social, normes, politique de l'emploi, rationalité

Keywords : incentives, labour law, norms, employment policy, rationality 


\section{Introduction}

Comment orienter les comportements des individus ? Cette question se trouve au cœur des réflexions sur les outils et l'efficacité des politiques publiques. À cet égard, un constat peut être dressé : une faveur nouvelle est aujourd'hui affichée au profit d'instruments de gouvernement des hommes qui encouragent, plutôt qu'ils ne contraignent. Les politiques de l'emploi offrent une belle illustration de la place prise par les dispositifs incitatifs, notamment les incitations financières de mise au travail ${ }^{1}$. Plus largement, les mesures concernant le travail, et particulièrement la qualité du travail, revêtent parfois un caractère incitatif ${ }^{2}$. Dès lors, si le droit du travail n'est pas soluble dans les incitations ${ }^{3}$, la diffusion, en son sein, d'une logique incitative peut être constatée.

Cette évolution de l'instrumentation juridique de l'action publique est laissée en bonne partie inexplorée ${ }^{4}$. Lorsqu'elle est pleinement affrontée, elle donne lieu à des appréciations contrastées. Les incitations, surtout financières, sont bien souvent réduites à un instrument économique, par opposition aux dispositifs contraignants qui relèveraient de la sphère juridique. Incitations économiques versus normes juridiques: le contraste est saillant et commode. À cette opposition - que nous discuterons par la suite - se trouve généralement associée une partition disciplinaire. L'étude des incitations, de l'opportunité de les mettre en place, de leurs effets, de leur paramétrage reviendrait aux économistes. L'analyse des dispositifs contraignants, de leur cohérence, de leur conformité avec leur environnement juridique serait, quant à elle, l’apanage des juristes.

Cette répartition repose sur un présupposé : celui du caractère a-juridique des incitations. Mais une telle opposition ne résiste pas à l'examen, ne serait-ce que parce que les dispositifs incitatifs prennent place dans des énoncés juridiques. Du reste, le Conseil constitutionnel n’hésite pas à se reconnaître compétent pour statuer sur la validité juridique d'un dispositif financier incitatif 5 .

Mais affirmer que les dispositifs incitatifs ${ }^{6}$ sont aussi des règles juridiques soulève une interrogation autrement délicate : les normes incitatives constituent-elles des règles juridiques d'un type particulier ? Ou, au contraire, faut-il considérer que toutes les règles juridiques ont un effet incitatif, et que, partant, il n’y a pas lieu de réserver le qualificatif « incitatif » à

\footnotetext{
${ }^{1}$ Le constat n'est pas nouveau. En ce sens, voir Piketty [1998].

${ }^{2}$ Pour des exemples, voir Blin-Franchomme, Desbarrats [2010].

${ }^{3}$ Référence est faite ici à la contribution de Frédéric Eymard-Duvernay parue dans la revue Droit social en 2004, [Eymard-Duvernay, 2004].

${ }^{4}$ Les mécanismes d'incitation font l'objet de mentions limitées dans des ouvrages de référence comme Boussagnet et al. [2004] ou Halpern et al. [2014].

${ }^{5}$ Peut être mentionnée la décision du 29 décembre 2009 dans laquelle le Conseil constitutionnel a censuré la taxe carbone (2009-599 DC).

${ }^{6}$ L'expression « dispositif incitatif » nous paraît la plus juste, car la plus générale. En effet, c'est le plus souvent un ensemble de règles qui produit l'effet incitatif sur les comportements, sans qu'il soit d'ailleurs toujours simple de délimiter l'étendue de cet ensemble de règles. Toutefois, il n'est pas exclu qu'une règle prise isolément présente également un caractère incitatif. Pour cette raison, nous prenons ici le parti de recourir aussi bien aux expressions « norme incitative » que « dispositif incitatif ».
} 
certaines d'entre elles ? C'est volontiers la deuxième affirmation qui a la faveur des juristes ${ }^{7}$ comme des économistes. La tentation est grande, en effet, de considérer toute règle juridique comme une incitation, la sanction dont la règle est assortie constituant un signal encourageant les individus à ne pas adopter le comportement réprimé. Dans cette perspective, toute règle peut, en définitive, être interprétée comment étant incitative.

Pourtant, l'observation des règles de droit en vigueur interdit de se laisser enfermer dans cette alternative, conduisant soit à opposer radicalement les règles contraignantes et les incitations, soit, à l'inverse, à les identifier l'une avec l'autre et les confondre. Car, en effet, ce double écueil ne permet pas de rendre intelligibles les changements à l'œuvre dans le droit du travail [Leroy-Arlaud, 2014]. Pour les comprendre, il nous semble nécessaire de faire l'hypothèse que certains dispositifs juridiques méritent, plus que d'autres, de se voir apposer l'épithète « incitatif ».

Seul un travail de spécification de ce qu' « incitatif » veut dire permet d'analyser les mutations de l'instrumentation juridique du droit du travail (§3). Mener à bien cette tâche suppose au préalable d'abandonner certaines distinctions qui sont présentes dans la littérature juridique, mais qui conduisent, en réalité, à obscurcir l’analyse (§2). Cela fait, il devient possible de mesurer les effets engendrés par les dispositifs incitatifs dans le champ du travail et de l'emploi. Il serait, en effet, réducteur d'analyser les mutations à l'œuvre comme le simple changement d'un moyen, d'un instrument n'affectant pas l'armature - notamment axiologique - du droit. Des exemples tirés aussi bien des conditions dans lesquelles se déroule le rapport d'emploi que du droit des relations professionnelles rendent sensibles les déplacements conceptuels qui accompagnent les changements de l'instrumentation juridique mise en œuvre. Car, en effet, La force d'irradiation de la logique incitative ne laisse indemnes ni le droit du travail ni les catégories juridiques qui structurent la relation de travail (§4).

\section{Des distinctions inopérantes}

Deux distinctions, pourtant courantes dans les écrits juridiques, s’avèrent inopérantes pour attribuer une signification juridique à l'adjectif « incitatif ».

La première porte sur la nature des actions suggérées par les règles juridiques : les incitations encourageraient à agir, tandis que les dispositifs contraignants viseraient à empêcher des comportements prohibés ${ }^{8}$. Ce partage est fragile. Les règles de droit peuvent, en effet, avoir les objets les plus divers [Jeammaud, 1990]. Les exemples ne manquent pas de règles qui imposent des comportements, introduisent des obligations de faire ou de donner. Que l'on pense au devoir de secours entre les époux ou, dans le cadre des relations du travail, à l'obligation de sécurité, aux obligations de négocier, à l'exigence d'égalité. Symétriquement, des dispositifs dits incitatifs peuvent parfaitement encourager l'inaction. Tel aurait été l'effet recherché par la taxe sur le licenciement, tant promue $^{9}$, si elle avait fait son

\footnotetext{
${ }^{7}$ Pour une conception de la sanction comme incitation, voir les analyses de la sanction pénale de C. Beccaria 1774 [2006].

${ }^{8}$ Chevallier [1993, p. 124] : « Des techniques d'incitation ont d'abord pris le relais des commandements juridiques. On a assisté au développement d'une direction juridique non autoritaire des conduites [...] procédant par voie de recommandations plutôt que de prescriptions. »
}

${ }^{9}$ En ce sens, voir Blanchard, Tirole [2003], Cahuc [2003], Cahuc, Kramarz [2004]. Pour une analyse 
entrée dans le droit positif. Vouée à remplacer l'encadrement procédural et substantiel dont fait l'objet le licenciement pour motif économique par le paiement d'une somme forfaitaire, une telle taxe visait à décourager la décision de licenciement, laquelle n’est adoptée que si elle est nécessaire. Dans cette perspective, c’est une abstention qui est encouragée.

Une seconde distinction, qui porte sur l'objet du dispositif juridique, mérite d'être discutée. Peut-on considérer qu'une incitation se caractérise par l'octroi d'une récompense, d'un avantage, tandis que la sanction attachée au non-respect d'une règle s'apparente nécessairement à un désagrément ? L'incitation n'est-elle que positive et la sanction serait-elle sa face négative ? Autrement dit, l'attribution d'un avantage est-elle un critère d'identification d'une norme incitative ? Ces interrogations peuvent être soulevées à propos des dispositifs de pénalisation des entreprises qui ont recours à des contrats à durée déterminée de très courte durée. À la suite de l'accord national interprofessionnel du 11 janvier 2013, le coût du recours aux contrats à durée déterminée pour un temps inférieur à trois mois a été renchéri ${ }^{10}$. Un tel dispositif doit-il être qualifié de sanction et non d'incitation ? Dans le prolongement des termes utilisés par un auteur, un tel dispositif pourrait être désigné comme " une mesure négative d'incitation » [Vacarie, 2005, p. 903].

À notre sens, l'incitation n'est pas la face positive de la sanction. Cette idée se décline à deux niveaux : toute conséquence légale positive (avantage) n'est pas une incitation (positive) ; inversement, une conséquence légale négative (sanction) peut être une incitation (négative). Autrement dit, l'attribution d'un avantage comme l'imposition d'une sanction peuvent être incitatifs. Nous rejoignons ici la pensée de Norberto Bobbio, qui s'attache à souligner le développement de la "fonction promotionnelle » du droit. L'un des caractères saillants du système juridique de l'État providence réside, selon lui, dans « l'augmentation des lois d'incitation, ou lois incitatives » [Bobbio, 1998, p. 75], qui recourent à la « technique de l'encouragement, consistant à promouvoir les comportements », quand un ordre juridique protecteur-répressif s'attache avant tout à décourager certains comportements en les réprimant. Pour autant, cette fonction promotionnelle peut être assurée, selon Norberto Bobbio, aussi bien par des sanctions positives que par des sanctions négatives ${ }^{11}$ : " Même si, de fait, les normes négatives sont habituellement renforcées par des sanctions négatives, et les sanctions positives principalement prévues et appliquées afin de renforcer les normes positives, il n'existe aucune incompatibilité entre normes positives et sanctions négatives d'un côté, et normes négatives et sanctions positives de l'autre » [Bobbio, 1998, p. 68].

En définitive, ce n'est ni le type d'action qu'il encourage ni sa substance promotionnelle ou sanctionnatrice qui justifient qu'un dispositif se voie associer l'épithète « incitatif ». C’est autrement qu'il convient de distinguer les incitations.

\section{Les attributs d'une incitation}

\footnotetext{
critique de ces rapports, voir Sachs [2013], Bargain [2014].

${ }^{10}$ Dans la continuité de l'accord national interprofessionnel du 11 janvier 2013, un avenant à la convention d'assurance chômage a introduit cette majoration depuis le $1^{\mathrm{er}}$ juillet 2013. Le principe de cette majoration a été réitéré dans la convention du $1^{\mathrm{er}}$ mai 2014 (art. 4).

${ }^{11}$ Bobbio [1998, p. 68] : « Il importe de ne pas confondre, d'un point de vue analytique, la distinction entre normes positives et normes négatives, et celle entre sanctions positives et sanctions négatives. »
} 
Distinguer, parmi les règles juridiques, celles qui méritent d'être considérées comme incitatives est un travail d'interprétation du droit, c’est-à-dire des énoncés juridiques ainsi que de leurs agencements. L'herméneute ne peut entreprendre un tel travail qu'à la condition d'être muni d'une grille d'interprétation, laquelle précise les attributs d'une norme incitative. Bref, on ne peut faire l'économie d'une définition de l'incitation, qu'il s'agira de construire aux fins de l'analyse.

Pour cela, le droit positif lui-même offre des ressources, lorsque la formulation des énoncés juridiques rend explicite le mécanisme incitatif. Il en est ainsi lorsque le Code du travail dispose que «l'État peut attribuer une aide financière du fonds de développement pour l'insertion professionnelle des handicapés à tout employeur soumis à l'obligation d'emploi de travailleurs handicapés afin de faciliter la mise ou la remise au travail en milieu ordinaire de production des personnes handicapées » (art. L. 5213-10) [Joly, 2015, n 526]. Ici, c’est la cohabitation de deux éléments dans un même énoncé qui retient l'attention : la formulation de l’objectif socio-économique recherché par la norme - « l'insertion des personnes en situation de handicap »- , d'une part, et la référence à une aide financière, d'autre part. Ce texte ne décrit pas le comportement vertueux ou sanctionné par la règle, mais précise les moyens octroyés à l'employeur pour atteindre un objectif social fixé par le législateur. La réunion de ces deux attributs fait explicitement place à une logique instrumentale - que rend sensible la préposition « afin de ».

Une finalité de la norme déterminée (§3.1.) et la technique d’attribution d’un avantage, qui peut passer aussi bien par l'octroi d'un bien que par l'évitement d’un mal (§3.2.) : retenons ces deux premiers attributs de l'incitation, qu'ils soient explicites ou non [LeroyArlaud, 2014]. Un troisième élément, qui autorise le passage d'une logique instrumentale à une logique incitative, est quant à lui nécessairement implicite. Il renvoie à la conception du destinataire de la règle de droit : un être rationnel et intéressé ${ }^{12}$ (§3.3.). La réunion de ces trois attributs, hétérogènes, caractérise une incitation ${ }^{13}$. Deux d'entre eux tiennent à la technologie juridique employée, tandis que le troisième réside dans la représentation que l'on se fait du destinataire de la règle.

\subsection{Une norme finalisée}

Les incitations se trouvent nécessairement orientées vers l'achèvement d'un objectif. Elles s'intègrent donc dans des programmes d'action, dont elles constituent l'une des modalités. Il n’y a dès lors pas lieu de s'étonner d'en trouver les principales manifestations dans les politiques publiques (politique de l'emploi, politiques d'insertion...) ${ }^{14}$. S'il n'est pas excessif de penser que l'adoption de toute règle juridique sert un ou plusieurs objectifs - et

\footnotetext{
${ }^{12}$ Sur la construction de l'homo œconomicus, voir Demeulanaer [2003].

${ }^{13}$ Pour une analyse approfondie de ce qui constitue un « dispositif » incitatif et ses incidences sur le droit social, voir les travaux précurseurs de Leroy-Arlaud [2014]. Cet auteur envisage un dispositif incitatif comme un ensemble de règles énonçant un comportement facultatif assorti d'une récompense.

${ }^{14}$ Norberto Bobbio voit dans la diffusion «de techniques d'encouragement qui viennent s'ajouter ou se substituer aux techniques de découragement " la marque de "l'action de l'État social contemporain » [Bobbio, 1998, p. 65].
} 
cela même si son identification est l'objet de controverses -, le statut de cet objectif s'avère bien particulier dans le cas des dispositifs incitatifs.

D’abord, le dispositif incitatif n’a de sens que rapporté à l'objectif extra-juridique qu'il sert (la diminution du chômage, l'insertion des personnes handicapées, l'insertion des jeunes, etc.). Cela tient à la nature essentiellement instrumentale de la règle incitative, laquelle trouve sa raison d'être dans l'objectif poursuivi. Ainsi, le renchérissement du recours aux contrats de travail à durée déterminée de très court terme n’a de sens que rapporté à sa finalité : dissuader le recours à ce type de contrat. Par contraste, l'article du code pénal qui sanctionne l'homicide volontaire assume une fonction - punir l'auteur de l'infraction indépendante de l'objectif extra-juridique d'éradication de comportements criminels qu'il sert également. Ainsi, dans un dispositif incitatif, la logique instrumentale prime, jusqu'à évincer ses autres raisons d'être.

Ensuite, dans un dispositif incitatif, il existe une très faible marge d'appréciation sur l'objectif servi par la règle. Celui-ci est fortement stabilisé, soit qu'il soit expressément énoncé ${ }^{15}$, soit que la réalisation du dispositif n’ouvre pas d'espaces de délibération sur les finalités poursuivies. Il y a là une autre manifestation de la prégnance d'une logique instrumentale. Tel est particulièrement le cas des dispositifs incitatifs formulés en chiffres. La mise en œuvre des incitations fiscales - au sens large du terme - requiert que soit vérifiée la réunion des conditions d'obtention de l'avantage fiscal, sans que ce travail ne conduise à s’interroger sur les finalités de la règle. De même, le dispositif qui renchérit le coût du contrat à durée déterminée de très court terme conduit seulement à constater la durée convenue pour le contrat et à en tirer les conséquences prévues par la loi ; la question des finalités de la règle devient indifférente, comme si une interprétation téléologique de la règle ne pouvait avoir lieu. Tel n’aurait pas été le cas si la règle avait été formulée différemment, prévoyant, par exemple, une pénalité pour les employeurs utilisant de manière abusive les contrats à durée déterminée de très court terme. Un débat judiciaire sur le sens de l'adjectif " abusif » aurait alors laissé ouvert un espace de discussion sur l'objectif poursuivi par la règle. Semblable clôture des délibérations sur les finalités ou les fonctions de la règle est d'autant plus remarquable que le droit du travail recèle de nombreux dispositifs qui se caractérisent par leur ambivalence [Lyon-Caen, 2004 ; Jeammaud, 2005]. L'article L. 1224-1 du Code du travail est à cet égard topique. La règle selon laquelle un transfert d'entreprise, qui répond à certaines conditions, laisse survivre les contrats de travail auprès du repreneur était à l'origine, en 1927, explicitement tournée vers la préservation de l'emploi. Avec le temps, ce dispositif a assumé de nouvelles fonctions, notamment faciliter l'externalisation, par les entreprises, de certaines de leurs activités. D'autres exemples, tels que l'évolution de la fonction de la reconnaissance de l'unité économique et sociale, ou encore la législation sur les contrats à durée déterminée, peuvent également être mentionnés. Les dispositifs incitatifs ne laissent pas de place à une telle ambivalence.

Enfin, la primauté de la logique instrumentale conduit à privilégier un registre d'évaluation de la règle de droit portant sur son efficacité, c'est-à-dire sa propension à atteindre les objectifs extra-juridiques qui lui sont assignés. Peu importe que la règle soit effective - c'est-à-dire que les agents aient adopté le comportement désiré [Jeammaud, Serverin, 1992] -, seule compte sa capacité à atteindre un objectif extra-juridique, socioéconomique.

\footnotetext{
${ }^{15}$ Voir l'article L. 5213-10 déjà mentionné. Toutefois, les exemples sont rares.
} 
Que l'atteinte de l'objectif soit prépondérante rejaillit sur le paramétrage de l'incitation. Dans son acception classique, la sanction mêle des considérations diverses : prévenir, réparer, punir [Van de Kerchove, 2005, p. 21]. Ainsi, déterminer une sanction, en particulier fixer son quantum lorsqu'elle présente un caractère financier, oblige à concilier des considérations tournées vers le passé (évaluation du préjudice et de la faute) et vers le futur. Par opposition, un dispositif incitatif se trouve exclusivement tourné vers la réalisation d'un objectif futur. En conséquence, son paramétrage, en particulier la détermination de son quantum financier, repose sur une appréciation des effets attendus et donc sur la compréhension des ressorts de l'action ${ }^{16}$.

Un exemple permet d'illustrer le propos. Dans le cadre d'un dispositif enjoignant le chef d'entreprise désireux de fermer un établissement rentable de céder ce dernier à un éventuel repreneur, le législateur avait prévu une amende s'élevant à 25 fois le SMIC par salarié licencié à la suite de la fermeture de l'établissement. Le Conseil constitutionnel a censuré cette disposition, estimant que cette amende était manifestement disproportionnée par rapport à la " faute » commise par le chef d'entreprise ${ }^{17}$. Ce faisant, les membres du Conseil se sont prononcés au regard du caractère punitif de la sanction, sans considérer son versant incitatif, pourtant relevé par le gouvernement dans ses observations. Aux termes de ces dernières, le montant choisi par le législateur avait pour but de placer le chef d'entreprise devant un choix : rechercher effectivement un repreneur pour l'établissement rentable dont la fermeture est envisagée ou payer l'amende destinée à la prise en charge par l'État des chômeurs occasionnés par cette décision. Ainsi, le montant choisi obéissait à une double logique de dissuasion et de responsabilisation.

Pour ces raisons, les normes incitatives se conçoivent, sous l'angle de la légistique ${ }^{18}$, comme des instruments juridiques qui doivent être correctement paramétrés pour produire les résultats attendus. Ce paramétrage consiste à fixer le quantum d'une incitation financière, ou encore à adopter d'autres formes d'incitations.

\subsection{La diversité des modes incitatifs}

Dans la continuité de l'approche proposée par Norberto Bobbio, Pierre-Emmanuel Berthier distingue deux types de dispositifs incitatifs : ceux par lesquels l'effet incitatif passe par la récompense et ceux par lesquels il passe par la facilitation [Berthier, 2015].

Les premiers revêtent le plus souvent une forme financière. Il en est ainsi, par exemple, des dispositifs qui associent à l'accomplissement d'une action l'octroi d'un avantage financier [Arnaud, 1993, $v^{\circ}$ "Incitation »]. En droit du travail, ils sont anciens ${ }^{19}$ et fréquents.

\footnotetext{
${ }^{16}$ Voir infra.

${ }^{17}$ Sur cette disposition (loi $n^{\circ}$ 2014-384 du 29 mars 2014 visant à reconquérir l'économie réelle) et la censure du Conseil constitutionnel (Cons. const. 27 mars 2014, $\mathrm{n}^{0}$ 2014-692 DC), voir notamment D. 2014. 1101, chron. J.-P. Chazal ; Fabre, 2014 ; Sachs, Vernac, 2014.

${ }^{18}$ Le mot « légistique » désigne «l'ensemble des règles, principes et méthodes qui doivent être observés dans la préparation des textes normatifs (lois, ordonnances, décrets, arrêtés) » (Guide de légistique, Paris, La Documentation française, 2007).

${ }^{19}$ Dès 1959 était prévue une exonération sociale et fiscale pour les sommes distribuées au titre d'un intéressement qui résultait de la signature d’un accord collectif (ord. nº 59-126 du 7 janvier 1959).
} 
À titre d'illustration, la mise en place du travail dominical par voie d'accord collectif est moins onéreuse que sa mise en place par décision unilatérale ${ }^{20}$. Ou encore l'État peut subventionner la mise en place de la gestion prévisionnelle des emplois ou des compétences ${ }^{21}$. Peuvent également être mentionnées les aides versées au titre de l'embauche d'un salarié en situation de handicap ${ }^{22}$. Dans ces exemples, l'adoption du comportement encouragé se concrétise par le bénéfice d'une récompense financière. Notons toutefois que le mécanisme juridique sur lequel repose cette récompense diffère dans chacun de ces cas de figure. S'agissant de la mesure visant à encourager l'emploi des travailleurs en situation de handicap, l'incitation repose sur un mécanisme éprouvé d'octroi d'une aide financière. En matière de travail dominical, le bénéfice de la récompense repose sur une articulation plus complexe des sources du droit du travail. La récompense - la non-application d'un minimum salarial découle du caractère subsidiaire de la règle étatique : la rémunération-plancher prévue par la loi ne s'applique que si un accord collectif n'a pas pu être conclu. Le comportement encouragé (conclure un accord collectif) est ainsi récompensé par la mise à l'écart de la règle légale.

À côté des dispositifs qui encouragent les comportements par l'octroi d'une récompense, peuvent être distingués ceux qui visent «à faciliter l'adoption de la conduite attendue » [Berthier, 2015]. C’est ainsi, par exemple, que la mise en place de la mobilité par voie d'accord collectif se trouve facilitée par la mise à l'écart des règles sur le licenciement pour motif économique ${ }^{23}$. Ici, l'effet incitatif ne passe pas par une récompense financière, mais par la mise à l'écart d'un cadre normatif moins favorable. Il en va de même, par exemple, des dispositions de l'article L. 1111-3 du Code du travail qui excluent les apprentis du calcul de l'effectif de l'entreprise : l'employeur est encouragé à embaucher des apprentis par la mise à l'écart des règles de calcul de l'effectif de l'entreprise, et par conséquent, en évitant de franchir certains seuils d'effectifs qui obligent à la mise en place d'institutions représentatives du personnel.

Qu'elles reposent sur une logique de récompense ou de facilitation, les incitations partagent un point commun : celui de reposer sur une certaine conception du destinataire de la règle.

\subsection{Un destinataire rationnel et intéressé}

Ce qui fonde le caractère incitatif de ces techniques est le fait qu'elles visent à orienter les actions des personnes en utilisant de manière instrumentale les ressorts (supposés) de leur action, en particulier la poursuite de l'intérêt financier individuel. En offrant un avantage financier en contrepartie de l'adoption d'un comportement donné, la règle oriente la personne vers ce comportement. Le gouvernement des personnes est ainsi assuré non plus par l'imposition de comportements présentés comme obligatoires, mais par l'orientation vers des comportements qu'il est de l'intérêt des personnes de choisir d'adopter. Au lieu d'imposer aux personnes de se conformer au modèle que la règle dessine (par exemple en l'assortissant

\footnotetext{
${ }^{20}$ Art. L. 3132-25-3 du Code du travail, lequel prévoit que le seuil de rémunération minimale ne s’applique qu'à défaut d'accord collectif.

${ }^{21}$ Art. D. 5118 du Code du travail.

${ }^{22}$ Art. L. 5213-10 du Code du travail.

${ }^{23}$ Art L. 2242-23 du Code du travail.
} 
d'une sanction), ses concepteurs s'efforcent d'assurer son effectivité future en ajustant la réalisation de ses fins aux ressorts qui sont supposés guider l'action spontanée des personnes visées par cette règle.

Les normes incitatives présentes dans le droit social se caractériseraient donc par le fait qu'elles s'appuient sur une théorie de l'action intéressée [Bargain 2014 ; Leroy-Arlaud 2014]. Ces règles sont conçues pour épouser les logiques spontanées d'action prêtées à ces acteurs, tout en réduisant ces dernières à la seule action intéressée ${ }^{24}$. La conception de l'agir qui est présupposée par la norme elle-même est celle de l’individu rationnel, qui agit dans la poursuite de son propre intérêt. Ses choix d'action sont donc nécessairement intéressés. De même, en situation d'information parfaite, il n'aura aucune hésitation sur le sens de l'action à entreprendre. En somme, l'individu visé par la règle est réputé se comporter comme un agent économique rationnel. La conception des comportements individuels sous-jacente est la suivante : «Les individus n’obéiront à la règle de droit que s’il est de leur intérêt de faire ainsi, et ils essaieront, en tout cas, de minimiser les désagréments que le droit leur impose » [Van den Bergh 1996, cité par Kirat 1998, p. 6]. Dès lors, une règle bien conçue doit prendre en compte ce ressort fondamental de l'orientation des personnes par référence à une règle de droit. On notera que l'usage des catégories de l'analyse économique classique à propos du droit se déplace et s'approfondit à cette occasion : il ne s'agit plus seulement d'évaluer ex post les règles de droit à la lumière de leur efficacité, mais de construire des dispositifs d'action qui intègrent dans leur conception même la rationalité économique prêtée à tout un chacun.

La conception de normes incitatives prend pleinement acte de ce que l'architecture normative peut se couler dans les ressorts de l'action individuelle plutôt que de la contraindre. L'efficacité de la règle n'est pas recherchée par l'imposition d'une conduite donnée sous contrainte, mais en raison de son architecture, qui semble s'appuyer sur les ressorts spontanés de l'action. En cela, les normes incitatives figurent, dans le répertoire du "paternalisme libertarien » [Thaler, Sunstein, 2003], aux côtés de dispositifs matériels [Thaler, Sunstein, 2010], pour susciter des actions en s'appuyant sur ce que les personnes doivent rationnellement vouloir. L'idée sous-jacente est que des règles incitatives doivent pouvoir conduire les individus à adopter certains comportements, non par la seule brutalité de l'exercice de la contrainte, mais en exploitant leur propension supposée à optimiser leur intérêt financier [Jolls, 2000 ; McGaughey, 2014 ; Alemanno, Sibony, 2015].

La réunion de trois attributs permet de distinguer un dispositif incitatif : il est finalisé ; il repose sur une technique de récompense ou de facilitation ; il postule une action guidée par l'intérêt. L'hétérogénéité de ces caractères est cruciale : un dispositif peut être dit incitatif, non seulement en raison de ses caractères propres - qui relèvent de la légistique et de la technique juridique -, mais aussi en considération d'une certaine représentation des rapports entre les règles et les actions. Le développement de l'incitation dans le domaine des rapports de travail apporte par conséquent des changements non seulement dans l'outillage du droit du travail, mais aussi dans la conception fondamentale des rapports qu'il régit.

\section{Des effets sur la teneur du droit du travail}

Il serait erroné de considérer la prolifération de normes incitatives comme un simple

\footnotetext{
${ }^{24}$ Pour une réflexion approfondie sur la diversité des ressorts de l'action, voir Thévenot [2006].
} 
changement de technologie juridique. Elles produisent au contraire des transformations profondes du droit positif, qui s'en trouve affecté non seulement dans sa technologie, mais aussi dans sa teneur. Nous nous bornerons à évoquer ici deux principales implications du recours aux incitations ${ }^{25}$ : une reconfiguration, le plus souvent implicite, du rapport d'emploi dans ses diverses dimensions, d'une part ; une articulation renouvelée de l'individuel et du collectif, d'autre part.

\subsection{Une reconfiguration du rapport d'emploi}

Conçu essentiellement comme un agent rationnel, le destinataire de la règle incitative se trouve dépourvu des attributs (sociaux) généralement attachés aux parties à la relation de travail. En particulier, l'employeur n'est alors pas considéré comme le détenteur d'un pouvoir, pas plus que le salarié n'est envisagé comme juridiquement subordonné. Témoigne de cet effacement du lien de subordination découlant du contrat de travail le phénomène de «bilatéralisation" des incitations : celles-ci peuvent être destinées à celui qui exerce le pouvoir, comme à celui qui y est soumis, à savoir le salarié [Berthier, 2015 ; Bargain, 2014].

Ainsi, en matière de travail dominical ${ }^{26}$, des dispositions légales encouragent le salarié à consentir à des « propositions » faites par l'employeur. Si le salarié reste libre de refuser de travailler le dimanche - son refus n'est pas constitutif d'une faute -, il se trouve néanmoins fortement incité à l'accepter par la majoration salariale qui lui est proposée. La logique du dispositif repose sur un présupposé : celui de l'existence d'une réelle capacité pour le salarié de choisir entre plusieurs possibilités offertes. Le salarié est alors moins vu comme une personne subordonnée, soumise à un pouvoir de direction, de contrôle et de sanction, que comme un être rationnel doué d'une pleine volonté, qu'il saura exercer au mieux de ses intérêts. Envisagé comme un être rationnel capable d'opérer les meilleurs choix pour luimême, le salarié se trouve retranché de tout collectif (la collectivité de travail) et semble n’être soumis à aucune pression, ni économique ni managériale. Largement irénique, cette conception du salarié et de sa volonté fait bien peu de cas de la subordination juridique, qui reste pourtant le critère déterminant pour identifier la qualité même de salarié.

Le recours à la technique incitative produit également des changements de grande ampleur sur la conception des prérogatives patronales. Il en est ainsi s'agissant de mesures incitatives, destinées aux employeurs, qui visent à orienter leurs décisions en matière d'emploi. A déjà été évoquée la proposition faite par quelques économistes de substituer à l'encadrement procédural et substantiel de la décision de rompre le contrat de travail pour motif économique une taxe sur le licenciement. À l'image du principe du pollueur-payeur, cette taxe posséderait selon ses promoteurs, une double qualité : socialement juste - parce qu'elle permet d'internaliser les externalités sociales de la décision patronale -, elle est également économiquement efficace, dans la mesure où les employeurs peuvent intégrer son montant dans leurs anticipations. Il n'en demeure pas moins qu'une telle mesure méconnaît une dimension majeure de la décision patronale de rompre le contrat de travail. Cette dernière ne peut être uniquement considérée comme une décision de gestion. Elle constitue également l'expression d'un pouvoir d'une personne privée sur une autre [Gaillard, 1985 ; Fabre, 2008].

\footnotetext{
${ }^{25}$ Pour une analyse élargie de l'impact des incitations sur le droit du travail et le droit de la sécurité sociale, voir aussi Ginon[2011] ; Serverin, Gomel [2012] ; Leroy-Arlaud [2014] ; Berthier [2015].

${ }^{26}$ Art. L. 3132-20 et s. du Code du travail.
} 
L'exigence qui pèse sur l'employeur de justifier la mesure qu'il prononce, en énonçant des motifs de licenciement, reflète cette dimension [Lyon-Caen, 2002]. Substituer une taxe à ce qui reste bel et bien une décision produit, sous couvert d'un changement technique, une transformation profonde de la conception même de ce que signifie décider pour l'employeur.

Une analyse similaire peut être proposée, s'agissant du renchérissement du recours aux contrats à durée déterminée introduit par la loi de sécurisation de l'emploi du 14 juin 2013. L'un des objectifs poursuivis par les promoteurs ${ }^{27}$ de ce dispositif était de favoriser un allongement de la durée des contrats à durée déterminée proposés par les entreprises. Cet objectif aurait pu être servi par des techniques juridiques déjà usitées dans la législation relative aux contrats à durée déterminée. Par exemple, dans le prolongement de l'énonciation de cas de recours licites à ce contrat déjà présents dans le Code du travail, il aurait été envisageable d'introduire des dispositions législatives limitant le recours à des contrats à durée déterminée de très court terme à certains cas (remplacement, accroissement temporaire d'activités, etc.). Telle n’a pas été la technique normative choisie. A été préféré un dispositif incitatif, celui du renchérissement. Ce faisant, la décision d'embauche d'un contrat à durée déterminée se trouve conçue principalement comme une décision de gestion. Qu'elle soit l'expression du pouvoir de direction économique de l'employeur se trouve ignoré. Voilà qui invite à appréhender les dispositifs incitatifs avec mesure : leur efficacité - qui reste dans bien des cas à établir - a un coût : l'affaiblissement corrélatif de l'exigence d'encadrement de l'exercice d'un pouvoir privé.

\subsection{Une articulation renouvelée de l'individuel et du collectif}

L'épanouissement d'une logique incitative en droit du travail renouvelle l'articulation des dimensions individuelles et collectives de la relation de travail. D'abord, l'hégémonie des objectifs auxquels se rapportent les incitations entraîne des perturbations dans la représentation même de la collectivité des salariés. Prenant place dans des politiques publiques, ces objectifs sont le plus souvent formulés à une échelle qui dépasse celle du cadre de travail, que ce soit l'établissement, l'entreprise, le groupe. Ce peut être un bassin d'emploi ou le pays dans son ensemble. À cet égard, l'objectif national d'amélioration de la situation d'emploi qui a présidé à l'adoption de la loi de sécurisation de l'emploi est symptomatique de la prégnance d'une représentation de la collectivité de travail déconnectée du cadre de travail, laquelle rejaillit sur les dispositifs adoptés ${ }^{28}$. En outre, ces objectifs tendent à fractionner la collectivité des salariés. Tel est le cas lorsque les dispositifs incitatifs sont orientés vers la promotion d'individus doués de certaines caractéristiques. Ils conduisent à établir des distinctions parmi les membres de la collectivité des salariés. Les incitations en faveur d'une meilleure insertion des personnes en situation de handicap ou des jeunes en sont une illustration. Ainsi, les dispositifs incitatifs, par la logique instrumentale qu'ils charrient, rendent insaisissable la collectivité des salariés ${ }^{29}$.

\footnotetext{
${ }^{27}$ On vise ici les partenaires sociaux qui ont conclu l'accord national interprofessionnel, qui à son tour a inspiré la loi.

${ }^{28}$ Sur l'évolution du droit du travail d'un droit de l'entreprise vers un droit du marché du travail, voir Sachs [2013].

${ }^{29}$ Sur le rôle du droit dans la constitution du collectif de travail, voir Borenfreund [1991].
} 
Outre les irradiations produites par la formulation d'objectifs, les moyens utilisés pour encourager la négociation collective d'entreprise renouvellent également l'articulation de l'individuel et du collectif. À l'évidence, la promotion de la négociation collective d'entreprise ne se confond pas avec celle d'une logique incitative. Mais ces deux tendances se croisent et se nourrissent l'une l'autre. D'une part, comme le notent certains auteurs, la diversification des domaines de la négociation collective d'entreprise - l'égalité hommesfemmes, la gestion des emplois et des compétences, l'insertion des personnes en situation de handicap, la mobilité interne, etc. - tend à l'ériger en instrument de politique publique [Peskine, 2014 ; Supiot, 2003 ; Jobert, 2013]. Les partenaires sociaux se trouvent parfois obligés, parfois encouragés, à s'emparer « d'objectifs qui dépassent leur intérêt propre » [Supiot, 2003, 67]. D’autre part se multiplient les mécanismes qui visent à encourager la négociation collective d'entreprise, notamment sur l'emploi, que ce soit dans le cadre des accords de mobilité ou des accords de maintien dans l'emploi. À la lecture des énoncés du Code du travail, il est malaisé de détecter sur quelle ingénierie repose le mécanisme incitatif. Pour l'employeur, l'incitation réside dans le bénéfice d'un régime du licenciement aménagé en cas de refus du salarié de se conformer à ce que prévoit l'accord collectif ; pour les salariés, l'incitation est implicite et non juridique : la préservation de l'emploi. Telle est du moins la ratio legis du dispositif, laquelle n’épuise pas son effet incitatif. En vérité, la possibilité pour les partenaires sociaux de s'emparer de ces thèmes existait déjà. Toutefois, les pouvoirs publics, en donnant à ces accords une appellation spécifique et en identifiant ainsi un objet singulier de négociation, entendent bien stimuler une négociation sur ce point. Ce faisant, « l'incitation par la nomination permet à la loi d'orienter le cours de la négociation, sans faire usage d'une obligation de négocier supplémentaire » [Peskine 2014, p. 438]. Et l'efficacité de ce type de dispositif - orienté vers des objectifs de politiques publiques, notamment la protection de l'emploi - suppose une atténuation des voix dissidentes. Aussi la promotion de ces incitations se traduit-elle par un affaiblissement de l'individu qui ne peut offrir de résistance individuelle ${ }^{30}$.

\section{Conclusion}

Ignorance du pouvoir patronal, fragilisation de l'individu salarié qui s'accompagne d'une valorisation de l'expression de sa volonté : autant de tendances qui témoignent des effets corrosifs des dispositifs incitatifs, condamnant ainsi une approche purement instrumentale des incitations. En cela, elles participent bien du processus de «politisationdépolitisation » à l'œuvre dans les processus d'élaboration et de mise en œuvre des politiques publiques [Halpern et al., 2014, p. 46]. Ces dernières ne sauraient être pensées comme des équivalents fonctionnels aux normes auxquelles elles pourraient se substituer. Les mutations qui affectent l'instrumentation du droit du travail sont porteuses de profondes révisions des équilibres normatifs à l'œuvre en son sein. La prégnance d'un gouvernement par les incitations ne peut donc être pensée indépendamment d'une analyse approfondie des déplacements conceptuels qu'il charrie. Ce constat signe-t-il une condamnation sans appel des incitations ? Il invite plutôt à discuter les soubassements ontologiques, moraux et politiques de cette technologie de gouvernement des hommes, notamment la représentation des individus qu'elle charrie.

\footnotetext{
${ }^{30}$ Sur l'affaiblissement de la faculté de refus des salariés, voir Borenfreund 2013.
} 


\section{Bibliographie}

Alemanno A., Sibony A.-L. (eds.) (2015), Nudge and the Law. What Can EU Law Learn From Behavioural Sciences?, Hart Publ.

ARnaud A.-J. (dir.), Dictionnaire encyclopédique de théorie et de sociologie du droit, Paris, LGDJ.

BARGAin G. (2014), Normativité économique et droit du travail, Paris, LGDJ, coll. « Droitéconomie ».

BECCARIA C. (1774 [2006]), Des délits et des peines, Flammarion, coll. « GF ».

Berthier P.-E. (2015), «Les incitations légales », Semaine sociale Lamy, supplément, $\mathrm{n}^{\circ}$ 1680, 2015, pp. 36-42.

Blanchard O., Tirole J. (2003), Protection de l'emploi et procédures de licenciement, rapport du Centre d'analyse économique, Paris, La Documentation française.

Blin-Franchomme M.-P., Desbarrats I., Droit du travail et droit de l'environnement, Lamy, coll. " Axe droit», 2010.BobBio N. (1998), Essais de théorie du droit, trad. M. Guéret, Paris LGDJ, coll. « La pensée juridique ».

BORENFREUND G. (2013), "Le refus du salarié face aux accords collectifs de maintien de l'emploi et de mobilité interne », Revue de droit du travail, p. 316.

BORENFREUND G. (1991), "La représentation des salariés et l'idée de représentation », Droit social, p. 685.

Boussaguet L., JACQuot S., RAVINET P. (dir.) (2004), Dictionnaire des politiques publiques, Paris, Presses de Sciences Po, coll. « Références ».

CAHUC P. (2003), Pour une meilleure protection de l'emploi, rapport au Centre d'organisation économique, CCIP.

CAHUC P., KRAmARz F. (2004), De la précarité à la mobilité : vers une sécurité sociale professionnelle, Paris, La Documentation française.

Chevallier J. (1993), «La juridicisation des préceptes managériaux », in Politiques et management public, vol. 11, $\mathrm{n}^{\circ} 4$, numéro spécial « Droit et management public » (préparé par Jean-Bernard Auby). p. 111-134.

Demeulenaere P. (2003), Homo œconomicus, Enquête sur la constitution d'un paradigme, Paris, Presses universitaires de France, coll. « Quadrige essais/débats ».

EYMARD-DUVERNAY F. (2004), «Le droit du travail est-il soluble dans les incitations ? ", Droit social, p. 812-816.

FABRE A. (2014), « La promesse présidentielle de "Florange” à l'épreuve du droit de propriété et de la liberté d'entreprendre », Constitutions, p. 204.

FABRE A. (2008), Le régime du pouvoir de l'employeur, Paris, LGDJ.

GAILLARD E. (1985), Le pouvoir en droit privé, Paris, Economica. 
GinON A.-S. (2011), « Démographie médicale et techniques juridiques d'incitation », Politiques et Management public, vol. 28, $\mathrm{n}^{\circ}$ 1, p. 13-25.

HAlPeRn Ch., LAscoumes P., LE GALÈS P. (dir.) (2014), L'instrumentation de l'action publique. Controverses, résistance, effets, Paris, Presses de Sciences Po, coll. « Gouvernances ».

JeAmmaud A. (2005), « Le droit du travail dans le capitalisme, question de fonctions et de fonctionnement ", in Le droit du travail confronté à l'économie, JEAMMAUD A. (dir.), Paris, Dalloz, coll. « Thèmes et commentaires », p. 15.

JEAmmaud A. (1990), « La règle de droit comme modèle », Recueil Dalloz, p. 199.

JeAmMAud A., SERVERIN E. (1992), « Évaluer le droit », Recueil Dalloz, p. 263.

JOBERT A. (2013), « La négociation d'entreprise dans la crise : innovations institutionnelles et sociales », Droit social, p. 323-338.

JoLLS Ch. (2000), « Behavioral Economic Analysis of Redistributive Legal Rules », in Cass. R. SunsteIn (ed.), Behavioral Law \& Economics, Cambridge University Press, p. 288.

Joly L. (2015), L'emploi des personnes handicapées, entre discrimination et égalité, Paris, Dalloz, coll. " Nouvelle bibliothèque des thèses ".

KIRAT Th. (1998), «La Law and Economics, le calcul et la règle », in A. JEAMMAUD et Th. KIRAT (coord.), " Le droit et l'économie : quelles rencontres ? », Maison Rhône-Alpes des sciences de l'homme, colloque des 28 et 29 sept, Lyon.

Leroy-Arlaud S. (2014), «Droit social et incitations. Contribution à l'étude des transformations de la normativité juridique », thèse université Paris Ouest - Nanterre La Défense.

LyON-CAEN A. (2002), « La procédure au cœur du droit du licenciement économique », Droit ouvrier, p. 161-164.

LyON-CAen G. (2004), "Permanence et renouvellement du droit du travail dans une économie globalisée », Droit ouvrier, p. 49.

McGaughey E. (2014), "Behavioural Economics and Labour Law », LSE Law, Society and Economy Working Papers 20/2014.

PESKINE E. (2014), « La célébration de l'accord collectif d'entreprise », Droit social, p. 438.

PIKETTY Th. (1998), L'impact des incitations financières au travail sur les comportements individuels : le cas français, Paris, La Documentation française.

SACHS T. (2013), « Vers un droit du marché du travail ? », Semaine sociale Lamy, n 1569.

SACHS T. (2013), La raison économique en droit du travail, LGDJ, coll. « Bibliothèque de droit social ».

SACHS T., Vernac S. (2014), «La fondamentalisation d'un ordre libéral. À propos de la décision du Conseil constitutionnel du 27 mars 2014, loi sur la reconquête de l'économie 
réelle », Revue de droit du travail, p. 528.

SERVERIN E., GOMEL B. (2012), « Le revenu de solidarité active ou l'avènement des droits incitatifs », Centre d'études de l'emploi, document de travail, n 154, p. 9.

Supiot A. (2003), « Un faux dilemme, la loi ou le contrat », Droit social, p. 59.

Thaler R. H., Sunstein C. R. (2003), « Libertarian Paternalism », The American Economic Review, vol. 93, n² 2, p. 175.

ThAleR R. H., Sunstein C. R. (2010 2008]), Nudge. La méthode douce pour inspirer la bonne décision, Paris, Vuibert.

ThÉVEnOt L. (2006), L’action au pluriel. Sociologie des régimes d'engagement, Paris, La Découverte.

VACARIE I. (2005), « Les tensions entre le droit de la santé et le droit de la sécurité sociale », Revue de droit sanitaire et social, $\mathrm{n}^{\circ}$ 6, p. 899.

VAN DE KERChOve M. (2005), "Les fonctions de la sanction pénale. Entre droit et philosophie », Informations sociales, vol. 7, n ${ }^{\circ} 7$. 